\title{
Quantum Hall effect in graphene: a functional determinant approach
}

\author{
C G Beneventano ${ }^{1}$ and E M Santangelo ${ }^{1}$ \\ Departamento de Física, Universidad Nacional de La Plata, Instituto de Física de La Plata, \\ UNLP-CONICET, CC 67, 1900 La Plata, Argentina \\ E-mail: gabriela@obelix.fisica.unlp.edu.ar and mariel@obelix.fisica.unlp.edu.ar
}

Received 22 October 2007, in final form 4 January 2008

Published 9 April 2008

Online at stacks.iop.org/JPhysA/41/164035

\begin{abstract}
We start the paper with a brief presentation of the main characteristics of graphene, and of the Dirac theory of massless fermions in $2+1$ dimensions obtained as the associated low-momentum effective theory, in the absence of external fields. We then summarize the main steps needed to obtain the Hall conductivity in the effective theory at finite temperature and density, with emphasis on its dependence on the phase of the Dirac determinant selected during the evaluation of the effective action. Finally, we discuss the behavior, under gauge transformations, of the contribution due to the lowest Landau level, and interpret gauge transformations as rotations of the corresponding spinors around the magnetic field.
\end{abstract}

PACS numbers: $11.10 . \mathrm{Wx}, 02.30 . \mathrm{Sa}, 73.43 .-\mathrm{f}$

\section{Introduction}

Graphene is a bidimensional array of carbon atoms, packed in a honeycomb crystal structure. Actually, each layer of a graphene sample can be viewed either as an individual plane extracted from graphite or as an array of unrolled carbon nanotubes. Quite unexpectedly, in 2004, stable monolayer samples of such material were obtained [1] and, in 2005, the Hall conductivity was measured in such samples, independently, by two groups [2]. More recently, a different behavior of the Hall conductivity was reported [3] for bilayer samples. The main difference between the behavior of the Hall conductivity of mono- and bi-layer samples is in the height of the jump around zero carrier density (or, equivalently, chemical potential).

From a theoretical point of view, the most remarkable feature of graphene is that, in a small momentum approximation, the charge carriers or quasi-particles behave as two 'flavors' (to account for the spin of the elementary constituents) of massless relativistic Dirac particles

1 Member of CONICET. 
in the two non-equivalent representations of the Clifford algebra (corresponding to the two non-equivalent vertices in the first Brillouin zone), with an effective 'speed of light' about two orders of magnitude smaller than $c$ [4].

In [5], we showed that a field theory calculation at finite temperature and density, based upon $\zeta$-function regularization of the Dirac determinant leads, in the zero-temperature limit, to a sequence of plateaux in the Hall conductivity consistent with the measured ones, each time the chemical potential goes through a nonzero Landau level. Moreover, it was shown in [6] that two of the three possible combinations of phases of the Dirac determinant in both nonequivalent Clifford representations predict a behavior around zero chemical potential consistent with the ones measured in mono- and bi-layer graphene. For a complete presentation of other approaches to the study of graphene see, for instance, [7], and references therein.

This paper presents, in section 2, a brief introduction to the structure of graphene, and to the derivation of the continuous Dirac effective theory, in the absence of external fields. Section 3 contains a review of our previous results on the subject, with emphasis on the role of the phase of the determinant in giving rise to different behaviors around zero chemical potential. In section 4, entirely new results are presented. In that section, we allow for complex chemical potentials, and concentrate on the contribution due to the lowest Landau level, in order to study the invariance of the effective action under large gauge transformations. By relating gauge transformations to rotations in the plane, we analyze the effect of a $2 \pi$ rotation for each of the three possible combinations of phases in both representations, and identify, in the zero-temperature limit, the resulting geometrical or Berry's phases. Our conclusions are presented in section 5 .

\section{Structure of graphene: effective continuous model}

In this section, we sketch the main steps leading to the effective Dirac model for graphene, in the absence of external fields. For more detailed presentations see, for instance, [4].

The structure of the direct lattice for graphene is shown in figure 1 . The direct lattice is a superposition of two triangular lattices, $A$ and $B$. The generators of lattice $A$ are $\mathbf{a}_{1}=\sqrt{3} a\left(\frac{1}{2}, \frac{-\sqrt{3}}{2}\right)$, and $\mathbf{a}_{2}=\sqrt{3} a\left(\frac{1}{2}, \frac{\sqrt{3}}{2}\right)$, where $a$ is the lattice spacing. The vectors $\mathbf{s}_{1}=a(0,-1), \mathbf{s}_{2}=a\left(\frac{\sqrt{3}}{2}, \frac{1}{2}\right)$ and $\mathbf{s}_{3}=a\left(\frac{-\sqrt{3}}{2}, \frac{1}{2}\right)$ connect each site in the lattice $A$ to its nearest-neighbor sites in the lattice $B$. The tight binding Hamiltonian can then be written as

$$
H_{0}=-t \sum_{\mathbf{r} \in \Lambda_{A}} \sum_{i=1}^{3}\left[a^{\dagger}(\mathbf{r}) b\left(\mathbf{r}+\mathbf{s}_{i}\right)+b^{\dagger}\left(\mathbf{r}+\mathbf{s}_{i}\right) a(\mathbf{r})\right],
$$

where $t$ is the uniform hopping constant.

In momentum space, with $\left\{\begin{array}{l}a(\mathbf{k}) \\ b(\mathbf{k})\end{array}\right\}=\sum_{\mathbf{r} \in \Lambda_{A}} \mathrm{e}^{-\mathrm{i} \mathbf{k} \cdot \mathbf{r}}\left\{\begin{array}{l}a(\mathbf{r}) \\ b(\mathbf{r})\end{array}\right\}$, it reads

$$
H_{0}=\sum_{\mathbf{k}}\left(\Phi(\mathbf{k}) a^{\dagger}(\mathbf{k}) b(\mathbf{k})+\Phi^{*}(\mathbf{k}) b^{\dagger}(\mathbf{k}) a(\mathbf{k})\right)
$$

with $\Phi(\mathbf{k})=-t \sum_{i=1}^{3} \mathrm{e}^{\mathrm{i} \mathbf{k} \cdot \mathbf{s}_{i}}$. After defining two-component spinors as $\psi(\mathbf{k}) \equiv(a(\mathbf{k})$, $b(\mathbf{k}))^{T}, \psi^{\dagger}(\mathbf{k}) \equiv\left(a^{\dagger}(\mathbf{k}), b^{\dagger}(\mathbf{k})\right)$, one obtains

$$
H_{0}=\left(\begin{array}{cc}
0 & \Phi(\mathbf{k}) \\
\Phi^{*}(\mathbf{k}) & 0
\end{array}\right) .
$$

$H_{0}$ vanishes at the six corners of the first Brillouin zone. Among these, only two are inequivalent, and can be chosen as

$$
\mathbf{k}=\mathbf{K}_{ \pm}= \pm\left(\frac{4 \pi}{3 \sqrt{3} a}, 0\right), \quad \Phi\left(\mathbf{K}_{ \pm}\right)=0 .
$$




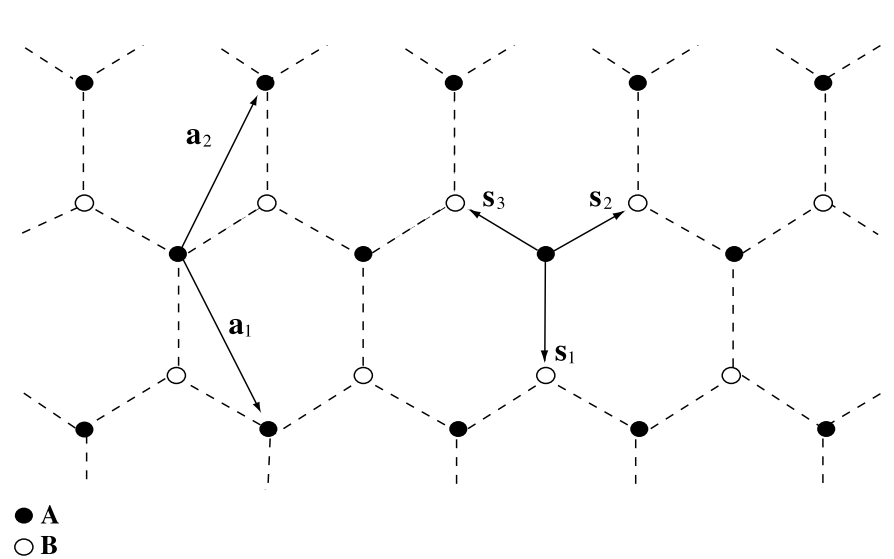

Figure 1. Direct lattice for graphene.

When $H_{0}$ is linearized around these two points, $\mathbf{k}=\mathbf{K}_{ \pm}+\mathbf{p}$, one obtains

$$
H_{\mathbf{k}=\mathbf{K}_{ \pm}+\mathbf{p}}=c\left(\begin{array}{cc}
0 & p_{x} \mp \mathrm{i} p_{y} \\
p_{x} \pm \mathrm{i} p_{y} & 0
\end{array}\right) .
$$

This last expression shows that each Fermi point gives rise to an effective Dirac theory, with an effective 'velocity of light' $c=\frac{3 t a}{2}$, in one of the two inequivalent representations of the gamma matrices. Thus, the total Hamiltonian can be taken as the direct sum of both (equivalently, as the Dirac Hamiltonian in the reducible 4 x 4 representation of the Clifford algebra). Moreover, an overall factor of two (two fermion species or 'flavors') must be included to take the spin of the original particles into account.

\section{The Hall conductivity from the interacting quantum field theory at finite temperature and density}

As shown in our previous work on the subject $[5,6]$, the Hall conductivity can be determined by first evaluating the partition function (equivalently, the effective action) for massless Dirac fermions at finite temperature and density, in two spacial dimensions, in the presence of an external magnetic field perpendicular to the plane, and then performing a boost to a reference frame with orthogonal electric and magnetic fields. In this section, we sketch our main results in those references, with emphasis on the role played by the phase of the Dirac determinant, which appears when treating the infinite tower of states associated with the lowest Landau level. We first consider a single flavor, and one of the two non-equivalent representations of the Clifford algebra.

In order to consider the effects due to finite temperature and density, we study the theory in Euclidean three-dimensional space, with a compact Euclidean 'time' $0 \leqslant x_{0} \leqslant \beta$, where $\beta=\frac{1}{k_{B} T}$ (here, $k_{B}$ is the Boltzmann constant and $T$ is the temperature). We introduce the (real) chemical potential and the magnetic field through a minimal coupling of the theory to an electromagnetic potential $A_{\mu}=\left(-\mathrm{i} \frac{\mu}{\mathrm{e}}, 0, B x_{1}\right)$. Natural units $(c=\hbar=1)$ will be used, unless otherwise stated.

In this scenario, the Euclidean effective action is given by $\log \mathcal{Z}=\log \operatorname{det}(\mathrm{i} \not \partial-e \not A)_{A P}$, where the subindex $A P$ indicates that antiperiodic boundary conditions must be imposed in 
the $x_{0}$ direction, in order to ensure Fermi statistics. Now, this is a formal expression, which we will define through a zeta-function regularization [8], i.e.,

$$
\left.\left.S_{\text {eff }}=\log \mathcal{Z} \equiv-\frac{\mathrm{d}}{\mathrm{d} s}\right\rfloor_{s=0} \zeta\left(s, \frac{(\mathrm{i} \not \partial-e \not A)_{A P}}{\alpha}\right)=-\frac{\mathrm{d}}{\mathrm{d} s}\right\rfloor_{s=0} \sum_{\omega}\left(\frac{\omega}{\alpha}\right)^{-s},
$$

where $\omega$ represents the eigenvalues of the Dirac operator acting on antiperiodic, squareintegrable functions, and $\alpha$ is a parameter introduced to render the zeta function dimensionless (as expected on physical grounds, our final predictions will be $\alpha$-independent).

So, in order to evaluate the partition function, we first determine the eigenfunctions, and the corresponding eigenvalues, of the Dirac operator. We propose

$$
\Psi_{k, l}\left(x_{0}, x_{1}, x_{2}\right)=\frac{\mathrm{e}^{\mathrm{i} \lambda_{l} x_{0}} \mathrm{e}^{\mathrm{i} k x_{2}}}{\sqrt{2 \pi \beta}}\left(\begin{array}{l}
\varphi_{k, l}\left(x_{1}\right) \\
\chi_{k, l}\left(x_{1}\right)
\end{array}\right) \quad \lambda_{l}=(2 l+1) \frac{\pi}{\beta} .
$$

Note that, in the last expression, $\lambda_{l}, l=-\infty, \ldots, \infty$ are the Matsubara frequencies adequate to the required antiperiodic conditions, while the continuous index $k$ represents an infinite degeneracy in the $x_{2}$ direction.

The resulting spectrum has two pieces, an asymmetric piece, associated with the lowest Landau level of the Hamiltonian:

$\omega_{l}=\tilde{\lambda}_{l}, \quad$ with $\quad \tilde{\lambda}_{l}=(2 l+1) \frac{\pi}{\beta}+\mathrm{i} \mu \quad$ and $\quad l=-\infty, \ldots, \infty$,

with corresponding eigenfunctions

$$
\psi_{k, l}\left(x_{1}\right)=\left(\begin{array}{c}
\left(\frac{\mathrm{e} B}{\pi}\right)^{\frac{1}{4}} \mathrm{e}^{-\frac{\mathrm{e} B}{2}\left(x_{1}-\frac{k}{\mathrm{e} B}\right)^{2}} \\
0
\end{array}\right)
$$

and a symmetric piece

$$
\omega_{l, n}= \pm \sqrt{\tilde{\lambda}_{l}^{2}+2 n e B} \quad \text { with } \quad n=1, \ldots, \infty \quad l=-\infty, \ldots, \infty,
$$

corresponding to eigenfunctions with both components being different from zero. In all cases, the degeneracy per unit area is given by the well-known Landau factor, $\Delta_{L}=\frac{\mathrm{e} B}{2 \pi}$.

The asymmetric part of the spectrum is quite particular. In the first place, as seen from (3), the corresponding eigenfunction is an eigenfunction of the Pauli matrix $\sigma_{3}$, with eigenvalue +1 . The eigenfunction with the opposite 'chirality' was eliminated by the square integrability condition in $x_{1}$. Moreover, in the other non-equivalent representation, this part of the spectrum appears with the opposite sign. Such a transformation is equivalent to $\mu \rightarrow-\mu$. This is nothing but charge conjugation (which, for real $\mu$, is also equivalent to a parity transformation [9]). As we will discuss in what follows, this part of the spectrum is the one which requires the consideration of a phase of the determinant when evaluating the effective action.

Before going to such evaluation, it is interesting to note the invariance of the whole spectrum under $\mu \rightarrow \mu+\frac{2 \mathrm{i} k \pi}{\beta}$. This invariance is a natural one, since such transformations preserve antiperiodicity of the eigenfunctions and, thus, the Dirac statistics. They are nothing but the so-called large gauge transformations. The invariance of the effective action under such transformations is also required for topological reasons. We will discuss this point in more detail in section 4.

As is clear from (2), in evaluating the effective action, one must perform the analytic extension of the contributions to the zeta function coming from both pieces of the spectrum,

$$
\zeta_{1}(s, \mu)=\Delta_{L} \sum_{l=-\infty}^{\infty}\left[(2 l+1) \frac{\pi}{\alpha \beta}+\mathrm{i} \frac{\mu}{\alpha}\right]^{-s},
$$


and

$$
\zeta_{2}(s, \mu, e B)=\left(1+(-1)^{-s}\right) \Delta_{L} \sum_{\substack{n=1 \\ l=-\infty}}^{\infty}\left[\frac{2 n e B}{\alpha^{2}}+\left((2 l+1) \frac{\pi}{\alpha \beta}+\mathrm{i} \frac{\mu}{\alpha}\right)^{2}\right]^{-\frac{s}{2}}
$$

The analytic extension of $\zeta_{2}(s, \mu, e B)$ is quite standard, and it relies mainly on performing a Mellin transform and making use of the inversion properties of the Jacobi theta functions. A detailed presentation can be found in [5]. The final result for the contribution to the effective action coming from this piece is (always considering only one representation of the gamma matrices and one fermion species):

$S_{\text {eff }}^{I I}=\Delta_{L} \beta \sqrt{2 e B} \zeta_{R}\left(-\frac{1}{2}\right)+\Delta_{L} \sum_{n=1}^{\infty} \log \left[\left(1+\mathrm{e}^{-(\sqrt{2 n e B}-\mu) \beta}\right)\left(1+\mathrm{e}^{-(\sqrt{2 n e B}+\mu) \beta}\right)\right]$.

The other non-equivalent representation of the Clifford algebra gives rise to an identical contribution, since this part of the spectrum is the same in both irreducible representations.

As said before, the extension of $\zeta_{1}(s, \mu, e B)$ requires a careful consideration of the phase of the determinant. In fact, $\zeta_{1}$ can be written as

$$
\zeta_{1}(s, \mu)=\Delta_{L}\left(\frac{2 \pi}{\alpha \beta}\right)^{-s}\left[\sum_{l=0}^{\infty}\left[\left(l+\frac{1}{2}\right)+\mathrm{i} \frac{\mu \beta}{2 \pi}\right]^{-s}+\sum_{l=0}^{\infty}\left[-\left(\left(l+\frac{1}{2}\right)-\mathrm{i} \frac{\mu \beta}{2 \pi}\right)\right]^{-s}\right],
$$

and the definition of the overall minus sign in the second sum depends on the selection of the cut in the complex plane of eigenvalues. As discussed in detail in [6], the usual prescription is to choose the cut in such a way that one does not go through vanishing arguments when continuously transforming eigenvalues with positive real part into eigenvalues with negative real part [10]. This prescription then gives rise to what will be called in the following the standard phase of the determinant (characterized from now on by $\kappa=-1$ ). One could certainly choose the opposite prescription, which we will call the nonstandard phase $(\kappa=+1)$. Once one of those phases is selected, the contribution of $\zeta_{1}$ to the effective action can be evaluated by making use of the well-known properties of the Hurwitz zeta function to obtain

$$
S_{\text {eff }}^{I}(\kappa)=\Delta_{L}\left\{\log \left[2 \cosh \left(\frac{\mu \beta}{2}\right)\right]+\kappa \frac{|\mu| \beta}{2}\right\} .
$$

When this last contribution is added to that in (4), one gets for the effective action

$$
\begin{aligned}
S_{\text {eff }}(\kappa)=\Delta_{L}\{ & \log \left[2 \cosh \left(\frac{\mu \beta}{2}\right)\right]+\kappa \frac{|\mu| \beta}{2}+\beta \sqrt{2 e B} \zeta_{R}\left(-\frac{1}{2}\right) \\
& \left.+\sum_{n=1}^{\infty} \log \left[\left(1+\mathrm{e}^{-(\sqrt{2 n e B}-\mu) \beta}\right)\left(1+\mathrm{e}^{-(\sqrt{2 n e B}+\mu) \beta}\right)\right]\right\} .
\end{aligned}
$$

From this last expression, the finite-temperature charge density can be obtained as $j_{0}(\kappa)=\frac{-\mathrm{e}}{\beta} \frac{\mathrm{d}}{\mathrm{d} \mu} \log \mathcal{Z}$. In the zero-temperature $(\beta \rightarrow \infty)$, and recovering physical units, it reduces to

$$
j^{0}\left(2 \mathrm{e} c^{2} \hbar B n<\mu^{2}<2 e B c^{2} \hbar(n+1)\right)=\frac{-\left(n+\frac{1+\kappa}{2}\right) c \mathrm{e}^{2} B}{h} \operatorname{sign}(\mu),
$$

where $n=\left[\frac{\mu^{2}}{2 \mathrm{e} B \hbar c}\right]$, and $[x]$ is the integer part of $x$. 

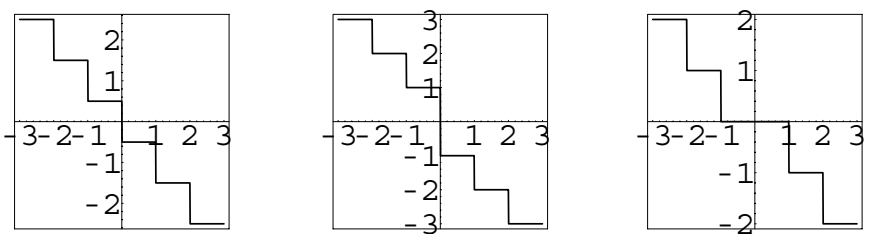

Figure 2. Hall conductivity for different selections of the phase of the determinant. Left to right: $K=1, K=2, K=0$. In all cases, the horizontal axis represents $\nu_{C}=\operatorname{sgn}(\mu) \mu^{2} / 2 \mathrm{e} B \hbar c^{2}$ and the vertical one, $\sigma_{x y} h / 4 \mathrm{e}^{2}$.

In order to obtain the Hall conductivity, one must perform a boost to a reference frame with crossed electric and magnetic fields. The final contribution to the Hall conductivity from each fermion species and one irreducible representation is given by [6] $\sigma_{x y}=\frac{-\left(n+\frac{1+\kappa}{2}\right) \mathrm{e}^{2}}{h} \operatorname{sign}(\mu)$.

Now, the phases of the determinant in both irreducible representations can be selected with the same or opposite criteria. When this is taken into account, and an overall factor of 2 is included to take both fermion species into account, one obtains for the total zero-temperature Hall conductivity

$$
\sigma_{x y}=\frac{-4\left(n+\frac{K}{2}\right) \mathrm{e}^{2}}{h} \operatorname{sign}(\mu),
$$

where $K=0$ corresponds to selecting the standard phase of the determinant in both irreducible representations, $K=1$ corresponds to choosing opposite criteria for the phases and $K=2$, to choosing both phases in the nonstandard way. The dependence of the Hall conductivity on the classical filling factor $\left(v_{C}\right)$ is presented in figure 2 for the three values of $K$. From this figure, it is clear that the behavior of monolayer graphene, as presented in [2], corresponds to $K=1$, i.e., to choosing opposite phases of the determinant in both representations (or, equivalently, ignoring the phase in both representations, as done in [11]). In fact, in this case the (rescaled) Hall conductivity shows a jump of height 1 for $v_{C}=0$, and further jumps of the same magnitude for $v_{C}= \pm 1, \pm 2, \ldots$ In turn, the behavior of bilayer graphene, as reported in [3] is exactly reproduced by $K=2$ (nonstandard selection of the phase in both representations).

\section{Invariance under large gauge transformations: interpretation in terms of rotations}

To analyze the physical meaning of the invariance of the effective action under large gauge transformations in this context, we go back to the zeta function associated with the asymmetric part of the spectrum, for two fermion species and one representation, this time allowing for an imaginary part in the chemical potential, $\tilde{\mu}=\mu+\mathrm{i} \gamma$, while always keeping $\mu \neq 0$. In this case, one must be careful when splitting the infinite sum as in (5) (detail of the calculations will appear in [12]). In fact, such splitting must be different for different $\gamma$-ranges, to make sure that all the eigenvalues in each infinite sum have a real part with the same sign, which is crucial in defining the phase. For example, for $-\frac{1}{2}<\frac{\gamma \beta}{2 \pi}<\frac{1}{2}$, one has

$$
\begin{gathered}
\left.S_{\text {eff }}^{I}\left(-\frac{1}{2}<\frac{\gamma \beta}{2 \pi}<\frac{1}{2}\right)=-2 \Delta_{L} \frac{\mathrm{d}}{\mathrm{d} s}\right\rfloor_{s=0}\left\{\sum_{l=0}^{\infty}[(2 l+1) \pi / \beta+\mathrm{i} \mu-\gamma]^{-s}\right. \\
\left.+\sum_{l=0}^{\infty} \mathrm{e}^{-\mathrm{i} s \theta}\left[(2 l+1) \pi / \beta+\mathrm{i}(\mu+\mathrm{i} \gamma) \mathrm{e}^{-\mathrm{i} \theta}\right]^{-s}\right\} .
\end{gathered}
$$

6 
Now, the values of $\theta$ such that the second term on the RHS does vanish are those ones for which, simultaneously, $(2 l+1) \pi / \beta+\mu \sin \theta-\gamma \cos \theta=0=\mu \cos \theta+\gamma \sin \theta$. As before, we consider here two different definitions of the phase of the determinant, which correspond to the standard definition for the phase $\kappa=-1$, and to the nonstandard one $\kappa=+1$. With each one of these prescriptions, the contribution of the asymmetric spectrum to the effective action is given by

$$
\begin{array}{r}
S_{\text {eff }}^{I}\left(-\frac{1}{2}<\frac{\gamma \beta}{2 \pi}<\frac{1}{2}\right)=2 \Delta_{L}\left\{\frac{(\kappa+1) \beta}{2} \operatorname{sgn} \mu(\mu+\mathrm{i} \gamma)\right. \\
\left.+\log \left(\mathrm{e}^{-\frac{\beta}{2}(\mu+\mathrm{i} \gamma)(1+\operatorname{sgn} \mu)}+\mathrm{e}^{\frac{\beta}{2}(\mu+\mathrm{i} \gamma)(1-\operatorname{sgn} \mu)}\right)\right\} .
\end{array}
$$

Things are entirely different for $\frac{\gamma \beta}{2 \pi}= \pm \frac{1}{2}$. In this case, one mode in the infinite sum defining the zeta function has a vanishing real part. A careful treatment shows that, at such points, $S_{\text {eff }}^{I}$ is discontinuous. For instance, $S_{\text {eff }}^{I}\left(\frac{\gamma \beta}{2 \pi}=+\frac{1}{2}\right)$ coincides with $\lim _{\frac{\gamma \beta}{2 \pi} \rightarrow \frac{1}{2}^{-}}$ of (7). An equally carefully treatment of the case $\frac{\gamma \beta}{2 \pi}=-\frac{1}{2}$ shows that $S_{\text {eff }}^{I}\left(\frac{\gamma \beta}{2 \pi}=-\frac{1}{2}\right)=$ $S_{\text {eff }}^{I}\left(\frac{\gamma \beta}{2 \pi}=\frac{1}{2}\right)$. This analysis can be extended to other ranges of variation of $\frac{\gamma \beta}{2 \pi}$, to obtain, with $k=-\infty, \ldots, \infty$,

$$
\begin{aligned}
S_{\text {eff }}^{I}\left(\left(k-\frac{1}{2}\right)\right. & \left.<\frac{\gamma \beta}{2 \pi} \leqslant\left(k+\frac{1}{2}\right)\right)=2 \Delta_{L}\left\{\frac{(\kappa+1) \beta}{2} \operatorname{sgn} \mu\left[\mu+\mathrm{i}\left(\gamma-\frac{2 k \pi}{\beta}\right)\right]\right. \\
& \left.+\log \left(\mathrm{e}^{-\frac{\beta}{2}\left(\mu+\mathrm{i}\left(\gamma-\frac{2 k \pi}{\beta}\right)\right)(1+\operatorname{sgn} \mu)}+\mathrm{e}^{\frac{\beta}{2}\left(\mu+\mathrm{i}\left(\gamma-\frac{2 k \pi}{\beta}\right)\right)(1-\operatorname{sgn} \mu)}\right)\right\} .
\end{aligned}
$$

This expression shows that the contribution to the effective action of the nonsymmetric part of the spectrum, in this representation of the gamma matrices, is invariant under large gauge transformations, no matter which phase of the determinant is selected. As already said, such transformations must constitute an invariance. In fact, an increase of i $\gamma$ in the chemical potential corresponds to the multiplication of the eigenfunctions (3) with a phase, i.e., $\psi_{k, l}(x) \rightarrow \mathrm{e}^{\mathrm{i} \gamma x_{0}} \psi_{k, l}(x)$. So, an increase $\mathrm{i} \gamma=\frac{2 \mathrm{i} \pi}{\beta}$ is a pure gauge transformation which, moreover, preserves the antiperiodicity in $x_{0}$.

Due to the fact that these eigenfunctions are eigenfunctions of $\sigma_{3}$ such that $\sigma_{3} \psi_{n=o}(x)=$ $\psi_{n=o}(x)$, one can equivalently write gauge transformations in the form $\psi_{k, l}(x) \rightarrow$ $\mathrm{e}^{\mathrm{i} \frac{\sigma_{3}}{2} 2 \gamma x_{0}} \psi_{k, l}(x)$. This last expression shows that, as $x_{0}$ grows from 0 to $\beta$, spinors are rotated by $2 \gamma \beta$, since $\frac{\sigma_{3}}{2}$ is the generator of rotations in the plane $x_{1} x_{2}$. In particular, $\gamma=\frac{2 \pi}{\beta}$ corresponds to a $4 \pi$-rotation around the magnetic field. Note that not only the partition function, but also the Abelian-Chern-Simons term (and, thus, the effective action) is invariant under large gauge transformations. On the other hand, $\gamma=\frac{\pi}{\beta}$ corresponds to a $2 \pi$-rotation. At finite temperature, such transformation changes the statistics to a bosonic one. For $\kappa=+1$, it also gives rise to an overall phase of $\pi$ per unit degeneracy in the partition function. Such a phase is the contribution which survives in the zero-temperature limit. The three possible combinations of phases of the determinant then give a total phase per unit degeneracy in the partition function of $\pi$ ( $K=1$, which reproduces the behavior of the Hall conductivity for monolayer graphene), or 0 (both for $K=0$ and $K=2$, this last reproducing the behavior of the Hall conductivity for bilayer graphene). At this point, it is interesting to note that, in order to have a zero-temperature partition function invariant under rotations of $2 \pi$ for monolayer graphene, the reduced flux through a unit cell of area $\Omega$ must be given by $\frac{\Phi}{\Phi_{0}}=\Omega \Delta_{L}=N$, with $N$ a positive integer. This is precisely the condition for physical states to transform as unidimensional ray representations of the magnetic translation group [9]. 


\section{Conclusions}

The first conclusion, as already stated in [6], is that two of the three possible combinations of phases give behaviors of the Hall conductivity which coincide with those measured in monoand bilayer graphene. In the case of bilayer graphene [3], the (rescaled) Hall conductivity presents a jump of height 2 for $v_{C}=0$, and further jumps of height 1 . The main point here concerns the positions of these subsequent jumps. As a matter of fact, according to figure $1(b)$ in [3], these subsequent jumps appear for $v_{C}= \pm 1, \pm 2, \ldots$, which is exactly the behavior predicted, in our calculation, for $K=+2$. However, the same reference interprets the Hall behavior of bilayer graphene through a theoretical prediction first made in [13] which, as discussed in [14], predicts a plateaux of larger width. Our calculation, instead, completely coincides with the measured behavior of the plateaux, both in height and width.

An entirely new conclusion is that, in each representation, the effective action per unit degeneracy is invariant under large gauge transformations, with any of the two possible selections of phase. As a result, the invariance persists no matter which of the three possible combinations of phases is selected. Moreover, each of the two selections of phase in each representation corresponds to a different geometric phase under the rotation of spinors along a closed path around the magnetic field $(\kappa=-1$ : no geometric phase; $\kappa=+1$ : geometric phase of $\pi$ ). So, different values of $K$ correspond to different total geometric phases per unit degeneracy, to be compared with the Berry phases studied, for instance, in [14]. Finally, by taking $\gamma \beta=\frac{\pi}{3}$, we note that, for $\frac{\Phi}{\Phi_{0}}=1$, these three values of $K$ also correspond to the three non-equivalent unitary representations of the generator of the cyclic group $C_{3}$, which is the relevant symmetry in the case of free graphene.

To the best of our knowledge, the relation between the phase of the fermionic determinant and Berry's phase had not been noticed before. This point, as well as the connection with the magnetic translation group will be studied in more detail in [12].

\section{Acknowledgments}

EMS thanks the organizers of QFEXT07 for the perfect organization, and for the nice atmosphere enjoyed during the event. This work was partially supported by Universidad Nacional de La Plata (Proyecto 11/X381) and CONICET (PIP 6160).

\section{References}

[1] Novoselov K S, Geim A K, Morozov S V, Jiang D, Zhang Y, Dubonos S V, Grigorieva I V and Firsov A A 2004 Science 306666

[2] Novoselov K S, Geim A K, Morozov S V, Jiang D, Katsnelson M I, Grigorieva I V, Dubonos S V and Firsov A A 2005 Nature 438197

Zhang Y, Tang Y-W, Stormer H L and Kim P 2005 Nature 438201

[3] Novoselov K S, McCann E, Morozov S V, Falko V I, Katsnelson M I, Zeitler U, Jiang D, Schedin F and Geim A K 2006 Nature Phys. 2 177-80

[4] DiVincenzo D P and Mele E J 1984 Phys. Rev. B 29 1685-94 Semenoff G W 1984 Phys. Rev. Lett. 532499

[5] Beneventano C G and Santangelo E M 2006 J. Phys. A: Math. Gen. 39 7457-70 Beneventano C G and Santangelo E M 2006 J. Phys. A: Math. Gen. 39 6137-44

[6] Beneventano C G, Giacconi P, Santangelo E M and Soldati R 2007 J. Phys. A: Math. Theor. 40 F435-42

[7] Neto A H Castro, Guinea F, Peres N M R, Novoselov K S and Geim A K 2007 Preprint 0709.1163

[8] Dowker J S and Critchley R 1976 Phys. Rev. D13 3224

[9] Dunne G V 1999 Topological Aspects of Low Dimensional Systems (Berlin: Springer)

[10] Cognola G, Elizalde E and Zerbini S 2003 Comm. Math. Phys. 237507 
[11] Schakel A M J 1991 Phys. Rev. D 43 1428-31

Peres N M R, Guinea F and Neto A H Castro 2006 Phys. Rev. B 73125411

Gusynin V P and Sharapov S G 2005 Phys. Rev. Lett. 95146802

[12] Beneventano C G, Giacconi Paola, Santangelo E M and Soldati Roberto 2007 (in preparation)

[13] McCann E and Fal'ko V I 2006 Phys. Rev. Lett. 96086805

[14] Luk'yanchuk I A and Kopelevich Y 2006 Phys. Rev. Lett. 97256801 